\title{
Comparing Agent Architectures in Social Simulation: BDI Agents versus Finite-State Machines
}

\author{
Carole Adam \\ Grenoble-Alps University, France \\ Grenoble Informatics Laboratory \\ Email: carole.adam@imag.fr
}

\author{
Patrick Taillandier \\ IDEES, Rouen University, France \\ Email: patrick.taillandier@univ-rouen.fr
}

\author{
Julie Dugdale \\ Grenoble-Alps University, France \\ Grenoble Informatics Laboratory \\ University of Agder, Norway \\ Email: julie.dugdale@imag.fr
}

\begin{abstract}
Each summer in Australia, bushfires burn many hectares of forest, causing deaths, injuries, and destroying property. Agent-based simulation is a powerful tool for decisionmakers to explore different strategies for managing such crisis, testing them on a simulated population; but valid results require realistic underlying models. It is therefore essential to be able to compare models using different architectures to represent the human behaviour, on objective and subjective criteria. In this paper we describe two simulations of the Australian population's behaviour in bushfires: one with a finite-state machine architecture; one with a BDI architecture. We then compare these two models with respect to a number of criteria.
\end{abstract}

\section{Introduction}

Each summer in Australia, bushfires burn many hectares of forest, causing deaths, injuries, and destroying property. Societies can manage such crisis and emergency situations in several ways: adopt urban and territory planning policies to reduce the risks (e.g. forbid construction in exposed areas); raise awareness and prepare the population in advance; or create efficient emergency management policies to deal with crises when they happen. Modelling and simulation offer tools to test the effects and complex interactions of these different strategies without waiting for an actual crisis to happen, without putting human lives at risk, with limited cost, and with a great degree of control on all conditions and the possibility of reproducing exactly the same situation as many times as needed.

When modelling human behaviour, mathematical, equation-based models are too limited [1]; on the contrary, agent-based models offer many benefits [2]. They allow capturing emergent phenomena that characterise such complex systems; they provide an intuitive and realistic description of their behaviour; they are flexible, offering different levels of abstraction by varying the complexity of agents. Agent-based modelling and simulation platforms offer various architectures of different complexity for the agents: reflex or reactive agents are very simplistic, reacting to environmental stimuli without any long-term reasoning; finite-state machines require scripting all of the possible states of the agents and the corresponding behaviours; cognitive agents offer a more flexible description of behaviours in terms of goals and plans.

In particular the BDI (belief, desire, intention [3]) architecture is very sophisticated and realistic, grounded in the philosophy of human rationality [4], and linked with emotions [5]. Such realism of the human behaviour model is important in order for the simulation to produce valid results [6]. The BDI architecture is therefore more adapted for crisis situations, that involve complex individual decision making, influenced by emotions (sometimes causing irrational actions), and by the social context (effect of group, family, etc.). BDI also provides the perfect level of abstraction to describe human behaviour in terms of folk psychology, which is the preferred level of description for humans [7]. It therefore addresses the problem of the scarcity of (quantitative) behavioural data by using qualitative data such as witness statements or expert reports. Despite these advantages making it very suitable for social simulation, BDI has had limited use in this field due to the lack of adapted tools to harness its complexity [8].

In previous work [9], [10], [11] we have described how two new tools could be used to develop BDI agent-based models from interviews. We illustrated how to use these tools by turning an existing model of the behaviour of the Australian population in bushfires ([12], with a finite-state machine architecture) into a BDI model. In this paper we want to compare these two models, addressing the same problem with different architectures, using both objective and subjective criteria. We believe such model comparison is important to further justify the interest of BDI models. This paper is structured as follows: in Section II we discuss the literature about model comparison. Section III introduces the case study and describes the two models in sufficient details. Section IV compares the simulation results of the two models with respect to the criteria discussed in Section II. Section V concludes the paper with a summary and discussion of future work.

\section{Model comparison}

In crisis management as well as other application fields of social simulation, many ad hoc simulators are created, with different agent architectures, different underlying models, and different tools; making comparisons hard. However 
model comparison is essential to determine which model is most appropriate in which application. In this section we discuss some existing work concerning model comparison.

\subsection{Existing work}

Model comparison has been the topic of many research works. Some of these works evaluate and compare the actual modelling platforms (e.g. Netlogo, Repast, Mason, etc.) [13], [14], [15], [16]. Others focus on comparing the performance of the resulting system. For instance [17] have compared the use of BDI and finite-state machines (FSM) architectures in games: evaluating complexity in terms of the number of behaviours, finding that it was linear for BDI agents and quadratic for FSM. On the other hand, run-time performance, using a small number of agents, degraded more quickly for BDI agents than for FSM agents in bigger systems.

Comparisons have also been made by focusing on the underlying models. In this context, most of works base their comparison on the simulation results and use two types of metrics: a fitness function - often computed by estimating the error between the observed data and simulated ones - and a computation time. For example, [18] compare three land-use change models based on three different architectures for the farmer agents: probabilistic, multi-criteria and BDI. The comparisons between the three models is achieved by using the fuzzy kappa coefficient [19] that allows evaluating the local similarity between the observed data and the simulated ones, and the percent absolute deviation.

Another technique that has been used by the agentbased community is "Docking", sometimes also known as "replication", or Model to Model comparison [20]. Docking attempts to align multiple models in order to investigate if they yield similar results. The compared models may all use an agent-based approach but be implemented in different platforms or languages for example [21], [22], [23], or they may use completely different approaches, by specifying their models using, for example, symbolic mathematical expressions or agents [24], [25]. The benefits of docking are well documented [22], [26] and include ensuring the validity of simulation results, increasing the quality of the model, and assessing if one model subsumes another.

Some works go further and propose measures to compare the complexity of models. Thus, [27], propose measures divided in three groups:

- Difficulty of description:

- number of parameters

- number of lines of code

- maximum cyclomatic complexity

- average cyclomatic complexity

- maximum nested block depth level

- average nested block depth level

- Difficulty of creation:

- computational time
- memory usage

- Difficulty of organization:

- approximate entropy

- fractal dimension

\subsection{Our comparison metrics}

This work aims at comparing two agent architectures through the comparison of two models. Contrarily to [27], the models will be very complex (integration of cognitive agents) and share many elements. As a result, some of the previously proposed metrics are not very well adapted. In addition, they do not propose any specific metric concerning the facility of appropriation of the models by users, which is a very important criterion for the re-usability of models.

We therefore propose the following metrics:

- Difficulty of description:

- number of characters in the code: we choose to use the number of characters rather than the number of lines as the length of lines can be very heterogeneous

- Difficulty of creation:

- computational time

- memory usage

- Difficulty of appropriation:

- understandability

- explanability

- extensibility

- Model credibility:

- error between observed data and simulated data

Note as stated by [28] the use of a modelling platform (such as Netlogo, GAMA or Repast) can facilitate model comparison. In this work we will therefore use the GAMA platform to compare the models.

\section{Simulating the population behaviour in Australian bushfires}

\subsection{Context}

We focus on the so-called Black Saturday, 7th February 2009, when particularly strong bushfires hit the state of Victoria in Australia, killing 173 people and destroying hectares of bush and many properties. The official recommendation to the population was to "prepare, stay and defend, or leave early". However, reports [29] written after these fires showed that emergency management policies were designed based on an (ideal) expected behaviour that differed from the residents' actual behaviour on the day. It is therefore important to provide deciders with a simulator to raise their awareness about residents' actual decision making, and let them try different communication strategies. Currently, the available data is mostly in the form of witness statements [30] and police hearings.

The research commission [31] also gathered the following statistics about the victims: 
- Preparation: $58 \%$ of the victims had made no preparation at all; many prepared to leave but expected a warning before going; $20 \%$ intended to stay and defend and were well prepared; $14 \%$ had made limited preparation.

- Awareness: the fire took by surprise $30 \%$ of those who died; $24 \%$ of the victims were unaware that they lived in a bushfire-prone area; $38 \%$ had no basic knowledge about what to do.

- Causes of death: $14 \%$ died while escaping (4\% by car and $10 \%$ by foot); $69 \%$ died while passively sheltering in a building; the others died while trying to defend.

- Vulnerability: $44 \%$ of the victims were considered vulnerable because of their age (under 12 or over 70 ), illness or disability; $32 \%$ died on properties whose defendability was questionable.

To facilitate comparison, we have implemented a general model in the GAMA simulation platform [32] that allows to choose between two models for the civilians' behaviour: the first one based on finite-state machine (FSM) and the second one on belief-desire-intention (BDI) .

Figure 1 presents the class diagram of the global model. For the sake of comparison, we defined a generic Civilian class that regroups all the common properties, actions and reflexes of both behaviour models.

Below we describe the model of the environment, buildings and fires; then the generic model of civilians and finally the two different models of civilians' behaviour. We try to give enough details to allow the reader to understand the model comparison provided in the next section.

\subsection{Model of the environment and the fire}

For the sake of simplicity, the environment is represented as a grid containing the different types of agents (houses, shelters, fires, and residents). This simplistic environment is not realistic but is sufficient to simulate the residents' decision-making behaviours in reaction to fires.

1) Fire.: Very complex and detailed models of fire spreading already exist [33], [34], but realistic fire behaviour is not the focus here. With the goal of not adding unnecessary complexity, we have designed a very simplistic fire model that is sufficient to trigger and visualise the reactions of the population in which we are interested. The fire is composed of fire agents (each with a location and an intensity representing its radius of action), having a reflex architecture, i.e. the following reflexes are triggered at each step of the simulation:

- Grow (Increase or decrease intensity): probabilities are parameters.

- Propagate to a non-burning neighbour cell, creating a new fire agent. Probability of propagating, and starting intensity of new fires, are parameters.

- Deal damage to buildings in its radius of action (based on its intensity): the amount of damage is picked randomly between 0 and a maximum value, function of intensity and a "damage factor" parameter.
- Deal injuries to residents in its radius of action, also a random amount between 0 and the maximum value based on its intensity and an "injury factor" parameter. If the person is in their house, the injury is moderated by its resistance weighed by a "protection factor" parameter.

- Disappear when its intensity is null.

The different parameters involved allow the user of the simulation to make the fire more or less dangerous in order to observe the desired behaviours. Actions are also available to start new fires or stop all fires (and thus the simulation).

2) Houses.: The environment initially contains a number (parameter) of houses each inhabited by exactly 1 resident (in future work we plan to consider families and their relationships). Each house is an agent with the following attributes:

- Owner: the resident of that house

- Resistance: random initial value between 100 and 200 to simulate different solidity. This value is increased by the resident preparing the house for a fire, or decreased by fire damage. Resistance offers some protection from fire injuries to its resident.

- Damage: the damage received from fire

The houses collapse from fire damage when their resistance drops to 0 . They then cease to offer protection, and the resident's motivation to defend them also disappears. Houses stay in the environment as ruins for final visualisation.

3) Shelters: are safe places offering total protection from the fires (no injuries can be received while in a shelter). Once a resident has reached a shelter, they stay inside until the end of the simulation.

\subsection{Generic civilian model}

Civilian agents are heterogeneous agents, each having their own values of attributes :

- defend motivation: random initial value between 0.0 and 1.0 to simulate the propensity to defend their home.

- escape motivation: random initial value between 0.0 and 1.0 to simulate the propensity to escape in case of danger.

- awareness probability: random initial value between 0.0 and 1.0 to simulate the attention towards dangers.

- perception radius: random initial value between 0.0 and 20.0 to simulate the maximal distance of perception of fires

- defense radius: random initial value between 0.0 and 2.0 to simulate the area of defense.

- danger radius: random initial value between 0.0 and 10.0 to simulate the area of danger.

- velocity: random initial value between 0.2 and 1.0 to simulate the moving speed.

- Location on the grid.

- House id (each agent is initially in a house). 


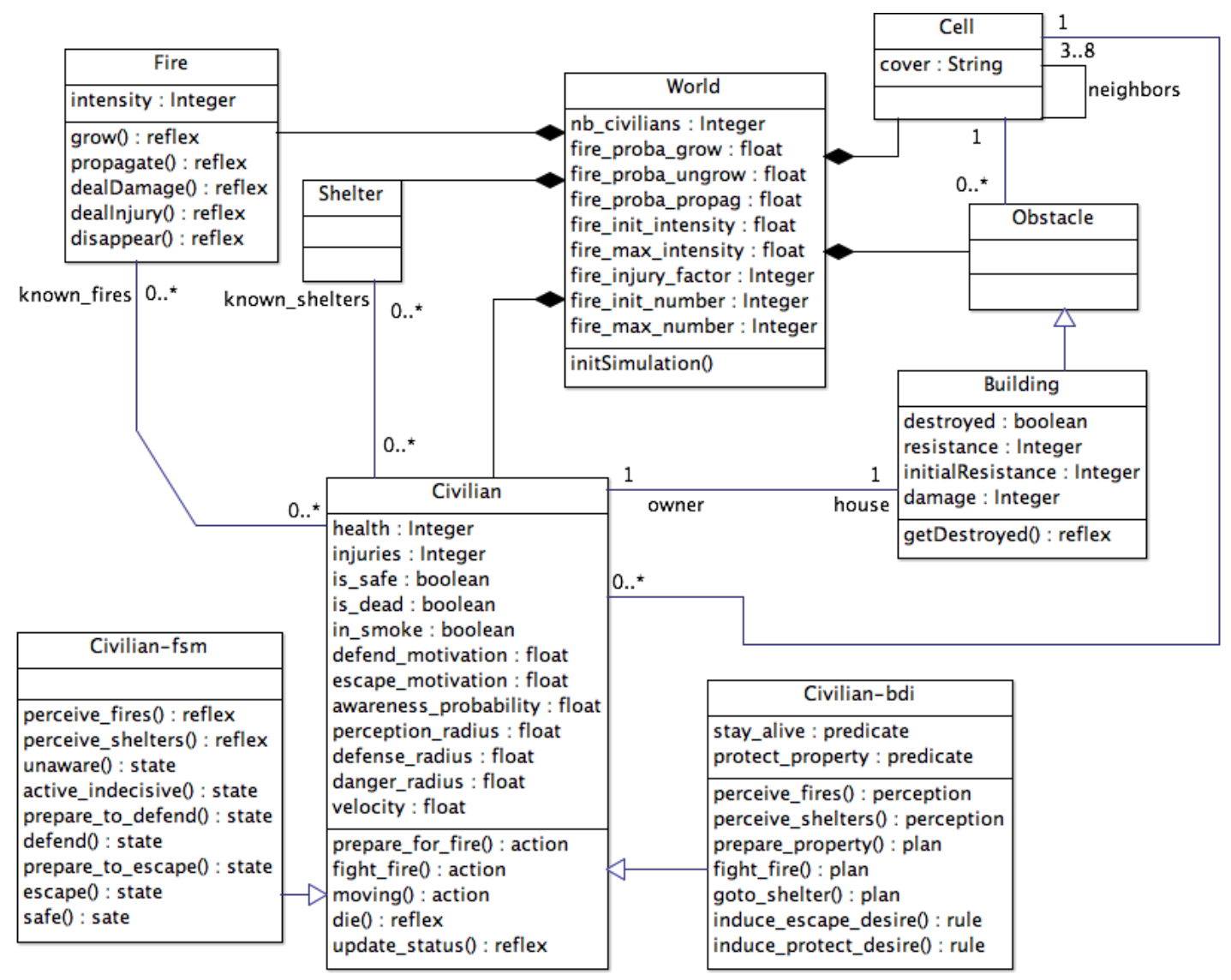

Figure. 1. UML Class diagram of the model

- health: the health of the civilian. It is increased by preparing for fire, decreased when receiving injuries. A health of 0 means death.

- injuries: the injuries received from fire. It decreases the health.

- is safe: defines if the civilian is in a shelter

- is dead: defines if the civilian is dead

- in smoke: defines if the civilian is in smoke (slow movement)

In addition, each civilian agent has the following actions:

- Prepare for fire: consists in increasing the resistance of the house (watering, weeding, etc.) and health (wearing appropriate clothing, etc).

- Fight fire: consists in decreasing the intensity of nearby fires by a value.

- Moving: consists in heading towards the closest shelter (amongst the known shelters); the agent might get injured if travelling too close to the fire.

Finally, they have reflexes:

- update status: is activated at every step; updates the agent speed according to the health and the presence of smoke; it also updates the agent's motivation to defend the property according to the agent context.
- die: is activated when the health of the agent reaches 0 ; makes the agent die (the attribute isdead is set to true).

\subsection{Finite-state machine simulation}

1) Conceptual model: Civilian-fsm agents have a finitestate-machine architecture with the following states and transitions (cf Figure 2):

- Unaware: initial state where the agent is (rightly or wrongly) unaware of any danger, and does nothing; agents can become aware by spotting fires in their perception radius (see flames, smell smoke, etc.), with a probability based on their objective abilities; they update their value of subjective danger based on their perceptions and motivations;

- Indecisive: the agent is aware of some fires but has not yet made a decision about how to react; agents stay indecisive for a varying amount of time, until they have enough motivation to either fight or escape; initial motivations are individual and then vary based on the evaluation of the situation (subjective danger);

- Preparing to escape: the agent has decided to leave and starts preparing, until ready or surprised by the fire before being ready (transition to Escaping), or blocked by the fire and forced to stay (transition to Preparing to defend);

- Escaping: the agent is evacuating towards the closest shelter (call the moving action); travel efficiency depends 
on objective abilities; injuries can be received from fires on the way. Unless the agent dies during travel, its next state will be Safe when reaching the shelter;

- Preparing to defend: the agent has decided to defend, or was forced to stay because the fire blocks escape; it prepares the house and itself (call the prepareforfire action) until the fire is close enough, which triggers the transition to Defending;

- Defending: the agent is actively fighting the fire around its house (call the fightfire action); when that fire is extinguished, the agent transitions back to Preparing to defend until another fire comes; if motivations change (e.g. subjective danger increases when actually seeing the fire, or subjective abilities decrease after failing to fight) and evacuation becomes more urgent, the agent transitions to Escaping;

- Safe: the agent is (and will stay) in a shelter, and cannot be injured anymore;

This simple model is sufficient to highlight the role of subjective, irrational determinants of the decisions and behaviours of each resident, and therefore captures the discrepancies shown by the data. Indeed the objective value of danger influences injuries and damage, and the objective value of capability influences the success of actions. But these objective values are inaccessible to the agents, whose decisions are based on their subjective values of danger and abilities, and on their motivations.

\subsection{BDI simulation}

We have later turned this initial FSM model into a BDI model.

In GAML (the programming language of the GAMA platform), the designer first needs to describe the different logical predicates that will be manipulated by the agent. This is basically the ontology of the domain. Agents can be endowed with an initial knowledge base with different beliefs and desires. These predicates can be associated with a priority. We define two predicates for the civilian-bdi agents:

- stay alive:desire to flee fires to stay alive. Its priority is based on the agent's danger aversion (escape motivation).

- protect property:desire to protect property. Its priority is based on the agent's danger determination (defend motivation).

Agents can then be given perceptions, that explain how they interpret the stimuli coming in. We define two perceptions for the civilian-bdi agents:

- perceive fires: perceive new fires (that are not yet in their list of known fires) and add them to their list.

- perceive shelters: perceive new shelters (that are not yet in their list of known shelters) and add them to their list.

Agents are also endowed with a number of rules. A rule allows inferring new beliefs or desires according to a specific condition (that can be a specific belief or desire). For the civilian-bdi agent, we define two rules:
- infer escape desire: infer the stayalive desire according to the awarenessprobability if the civilian knows that there is at least one fire (the agent refers to its known fires list).

- infer protect desire: infer the protectproperty desire according to the awarenessprobability if the civilian knows that there is a least one fire (known fires list).

The agents are endowed with a library of plans to achieve their goals. Each GAML plan is defined with several optional features: the goal that it achieves (keyword intention); a context condition (keyword when) that describes when this plan is applicable; and a success condition (keyword finished_when). We define three plans for the civilian-bdi agents:

- prepare property: it realises the goal protectproperty, by executing the prepareforfire action; it is applicable when the fire is not too close (i.e. the distance is higher than defense radius).

- fight fire: it realises the goal protectproperty, by executing the fightfire action; it is applicable when the fire is close enough (distance is lower than or equal to defense radius).

- go to shelter: it realises the goal stayalive, by executing the moving action.

\section{Comparing the simulations}

In this section, we compare the two simulations described in Section III with respect to the criteria discussed in Section II.

\subsection{Difficulty of description}

Table 1 shows the comparison of code length. We can observe that the code is far more compact with the BDI model (more than 24\% more compact). This is due to using specific features, like perceptions, that simplify writing the model, and to the fact that the FSM architecture requires explicitly specifying all the possible existing states and their transitions. This second explanation also shows a limitation of the FSM architecture in terms of modularity: enriching the model requires adding new states and specifying new transitions, which becomes increasingly complicated as the number of states increase, whereas adding new desires and plans in the BDI architecture is straightforward.

Table 1

CODE COMPARISON OF THE 2 MODELS (DIFFICULTY OF DESCRIPTION)

\begin{tabular}{|c|c|c|}
\hline Measure & FSM Model & BDI Model \\
\hline number of characters in the code & 1769 & 1310 \\
\hline
\end{tabular}

\subsection{Difficulty of creation}

Table 1 shows the comparison concerning the use of computer resources. For the time computation, we use the same scenario with 10 replications and the same series of seeds. The memory usage was estimated with 5000 civilian agents (and a grid of 100x100 cells) after 2 simulation steps 


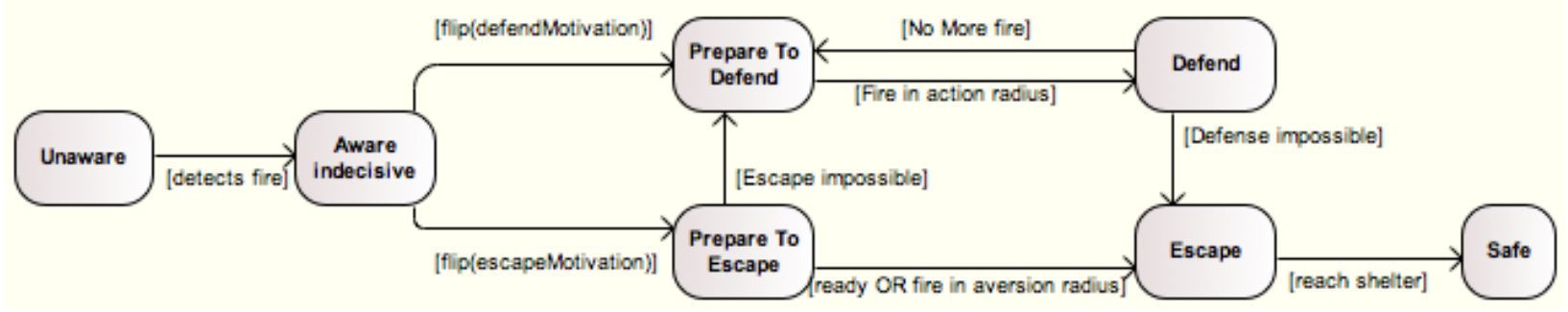

Figure. 2. States of the finite-state machine for Civilian agents

(the time for the agents to detect fires). The memory usage concerns the memory used by all aspects of the simulation, not only the civilian agents, but also all the other agents and the GAMA platform interface.

The computation times are relatively close. A deeper analysis with a profiling tool shows that the computation time is mostly due to the civilian agent actions and not to the computation linked to the architecture.

In the same way, the results for the memory usage are very close. Note that as our goal was to define a BDI model as close as possible as the FSM one, we did not use belief predicates that could have used more memory than basic variables (for example, for the known fires and known shelters).

Table 2

COMPARISON OF THE COMPUTER RESOURCE USAGE FOR THE 2 MODELS (DIFFICULTY OF CREATION)

\begin{tabular}{|c|c|c|}
\hline Measure & FSM Model & BDI Model \\
\hline Computation time & $35 \mathrm{~s}$ & $45 \mathrm{~s}$ \\
Memory usage & $103 \mathrm{Mo}$ & $109 \mathrm{Mo}$ \\
\hline
\end{tabular}

\subsection{Difficulty of appropriation}

We designed a questionnaire for test subjects to compare the two models on two aspects: understandability of code (can they understand how it works and modify it), and explanability of behaviour (can they understand what the agents do and why). We asked a limited number of testers (students from a computer science laboratory) to answer this questionnaire. These are more subjective criteria so this survey only provides qualitative feedback on the models. The testers found the BDI model more understandable and had less difficulty modifying it than the FSM model. It was also easier to explain behaviour in terms of what the agents desired instead of which state they were currently in. In future work we will conduct the study on a larger scale.

\subsection{Model Credibility}

In order to evaluate the model credibility, we used some of the data provided in III concerning bushfires, in particular the ones related to the cause of death:

- $14 \%$ died while escaping

- $69 \%$ died while passively sheltering in a building;
- $17 \%$ died while defending their property.

As the models are stochastic, we carried out 10 replications for each model with the same series of seeds.

Table 3 shows that the two models produce correct results even if they could be improved by enriching them to better take into account the heterogeneity of the behaviours. The difference between the results of the two models is not really significant. This was predictable as the Civilianbdi and Civilian-fsm agents share all their attributes and actions.

Table 3

COMPARISON OF THE MODEL OUTPUTS (MODEL CREDIBILITY)

\begin{tabular}{|c|c|c|c|}
\hline Measure & Real data & FSM Model & BDI Model \\
\hline Died escaping & $14 \%$ & $18 \%$ & $13 \%$ \\
Died passively & $69 \%$ & $72 \%$ & $68 \%$ \\
Died defending & $17 \%$ & $10 \%$ & $19 \%$ \\
\hline
\end{tabular}

\section{Conclusion}

In this paper, we discussed the need to compare agentbased models for social simulations using both objective and subjective criteria, in order to help designers determine which agent architecture is the most adapted for their simulation. Concretely, we focused on modelling the behaviour of the Australian population in bushfires, with two very different agent architectures: finite-state machines, and belief-desire-intention agents. We then compared these two models objectively on a number of criteria, and asked a number of subjects to subjectively compare their understandability and explainability. Our results show that BDI models, despite being more complex to program, offer a gain in modularity, flexibility and understandability. This is essential in crisis management where the goal of such models is precisely to explain behaviour, raise awareness, and explore new strategies.

In future work, we will further evaluate the agents models and increase the realism of the fire model, which has now become a limiting factor for observing realistic behaviours. Our long-term goal is to turn our simulation into a serious game for decision-makers.

\section{Acknowledgments}

This work was supported by the ANR ACTEUR project and AGIR SWIFT project. 


\section{References}

[1] H. Parunak, R. Savit, and R. Riolo, "Agent-based modeling vs equation-based modeling: A case study and users' guide," in MultiAgent Systems and Agent-Based Simulation, ser. LNAI. Springer, 1998, pp. 10-25.

[2] E. Bonabeau, "Agent-based modeling: Methods and techniques for simulating human systems," Proceedings of the National Academy of Sciences, vol. 99, no. 3, pp. 7280-7287, 2002.

[3] A. Rao and M. Georgeff, "Modeling rational agents within a BDIarchitecture," in 2nd International Conference on Principles of Knowledge Representation and Reasoning, 1991, pp. 473-484.

[4] M. E. Bratman, Intention, Plans, and Practical Reason. CSLI Publications, 1987.

[5] C. Adam, A. Herzig, and D. Longin, "A logical formalization of the OCC theory of emotions," Synthese, vol. 168, 2009.

[6] T. van Ruijven, "Serious games as experiments for emergency management research: A review," in 8th International ISCRAM Conference, Lisbon, Portugal, May 2011, need for realism of underlying model to get valid results, transferable to real life situations.

[7] E. Norling, "Folk psychology for human modeling: extending the BDI paradigm," in $A A M A S$, New York, 2004.

[8] C. Adam and B. Gaudou, "Bdi agents in social simulations: a survey," Knowledge Engineering Review, p. in press, 2016.

[9] C. Adam, G. Danet, J. Thangarajah, and J. Dugdale, "Bdi modelling and simulation of human behaviours in bushfires," in ISCRAM-med, 2016.

[10] C. Adam, E. Beck, and J. Dugdale, "Modelling the tactical behaviour of the australian population in a bushfire," in ISCRAM-med, ser. LNBIP. Springer, 2015

[11] "A situated bdi agent architecture for the gama modelling and simulation platform," in MABS workshop @ AAMAS. ACM, 2016.

[12] C. Adam and B. Gaudou, "Modelling human behaviours in disasters from interviews: application to melbourne bushfires," in Social Simulation Conference (SSC), Rome, Italy, September 2016.

[13] S. F. Railsback, S. L. Lytinen, and S. K. Jackson, "Agent-based simulation platforms: Review and development recommendations," Simulation, vol. 82, no. 9, pp. 609-623, 2006.

[14] M. Laclavík, Š. Dlugolinskỳ, M. Šeleng, M. Kvassay, B. Schneider, H. Bracker, M. Wrzeszcz, J. Kitowski, and L. Hluchỳ, "Agent-based simulation platform evaluation in the context of human behavior modeling," in Advanced Agent Technology. Springer, 2011, pp. 396-410.

[15] E. Daudé and P. Langlois, "Comparison of three implementations of schelling's spatial segregation model," Agent-Based Modelling and Simulation in the Social and Human Sciences, pp. chap-13, 2007.

[16] K. Bajracharya and R. Duboz, "Comparison of three agent-based platforms on the basis of a simple epidemiological model (wip)," in Proceedings of the Symposium on Theory of Modeling \& SimulationDEVS Integrative M\&S Symposium. Society for Computer Simulation International, 2013, p. 7.

[17] A. Bartish and C. Thevathayan, "Bdi agents for game development," in $A A M A S, 2002$.

[18] Q. C. Truong, P. Taillandier, B. Gaudou, M. Q. Vo, T. H. Nguyen, and A. Drogoul, Exploring Agent Architectures for Farmer Behavior in Land-Use Change. A Case Study in Coastal Area of the Vietnamese Mekong Delta. Cham: Springer International Publishing, 2016, pp. 146-158. [Online]. Available: http://dx.doi.org/10.1007/978-3-319-31447-1_10
[19] A. Hagen, "Fuzzy set approach to assessing similarity of categorical maps," International Journal of Geographical Information Science, vol. 17, no. 3, pp. 235-249, 2003.

[20] K. M. Carley, "Computational organizational science and organizational engineering," Simulation Modelling Practice and Theory, no. 5-7, pp. 253-269, 2002.

[21] R. Axtell, R. Axelrod, J. M. Epstien, and M. D. Cohen, "Aligning simulation models: A case study and results," Computational and Mathematical Organization Theory, vol. 1, pp. 123-141, 1996.

[22] S. N. Arifin, G. Davis, and Y. Zhou, "Verification and validation by docking: a case study of agent-based models of anopheles gambiae," in Summer Computer Simulation Conference, 2010, pp. 236-243.

[23] X. Xiang, R. Kennedy, and G. Madey, "Verification and validation of agent-based scientific simulation models," in Agent-Directed Simulation Symposium (ADS), 2005.

[24] M. North and C. Macal, "The beer dock: Three and a half implementations of the beer distribution game," in SwarmFest. Swarm Development Group, 2002.

[25] S. Rank, "Docking agent-based simulation of collective emotion to equation-based models and interactive agents," in Spring Simulation Multiconference. Society for Computer Simulation International, 2010, p. 6.

[26] J. Rouchier and E. Tanimura, "Learning with communication barriers due to overconfidence. what a "model-to-model analysis" can add to the understanding of a problem," Journal of Artificial Societies and Social Simulation, vol. 19, p. 7, 2016. [Online]. Available: http://jasss.soc.surrey.ac.uk/19/2/7.html

[27] A. Mandes and P. Winker, "Complexity and model comparison in agent based modeling of financial markets," Joint Discussion Paper Series in Economics, Tech. Rep., 2015.

[28] B. Müller, S. Balbi, C. M. Buchmann, L. De Sousa, G. Dressler, J. Groeneveld, C. J. Klassert, Q. B. Le, J. D. Millington, H. Nolzen et al., "Standardised and transparent model descriptions for agentbased models: current status and prospects," Environmental Modelling \& Software, vol. 55, pp. 156-163, 2014.

[29] Alan Rhodes, "Why don't they do what we think they should?" in AFAC. Emergency Management Victoria, 2014.

[30] S. Exell, "Witness statements," http://vol4.royalcommission.vic.gov. au/index03a1.html?pid=111.

[31] B. Teague, R. McLeod, and S. Pascoe, "Final Report, Volume I, Part 2: The people who died. Chapter 21: lessons learnt." 2009 Victorian Bushfires Royal Commission, Tech. Rep., 2009. [Online]. Available: http://goo.gl/0xcUFG

[32] A. Grignard., P. Taillandier, B. Gaudou, N. Huynh, D.-A. Vo, and A. Drogoul, "Gama v. 1.6: Advancing the art of complex agent-based modeling and simulation," in PRIMA, 2013.

[33] T. J. Duff, D. M. Chong, and K. G. Tolhurst, "Quantifying spatiotemporal differences between fire shapes: Estimating fire travel paths for the improvement of dynamic spread models," Environmental Modelling and Software, vol. 46, pp. 33-43, 2013.

[34] "Spark: A bushfire spread prediction tool," Environmental Software Systems. Infrastructures, Services and Applications, vol. 448, pp. 262-271, 2015. 